\title{
Radius measurement in binary stars: simulations of intensity interferometry
}

\author{
Km Nitu Rai, ${ }^{1 \star}$ Soumen Basak, ${ }^{1}$ and Prasenjit Saha ${ }^{2}$ \\ ${ }^{1}$ School of Physics, Indian Institute of Science Education and Research Thiruvananthapuram, Maruthamala PO, Vithura, \\ Thiruvananthapuram 695551, Kerala, India \\ ${ }^{2}$ Physik-Institut, University of Zurich, Winterthurerstrasse 190, CH-8057 Zurich, Switzerland
}

\begin{abstract}
Mass and radius measurements of stars are important inputs for models of stellar structure. Binary stars are of particular interest in this regard, because astrometry and spectroscopy of a binary together provide the masses of both stars as well as the distance to the system, while interferometry can both improve the astrometry and measure the radii of the stars. In this work we simulate parameter recovery from intensity interferometry, especially the challenge of disentangling the radii of two stars from their combined interferometric signal. Two approaches are considered: separation of the visibility contributions of each star with the help of differing brightness ratios at different wavelengths, and direct fitting of the intensity correlation to a multi-parameter model. Full image reconstructions is not attempted. Measurement of angular radii, angular separation and first-order limb-darkening appears readily achievable for bright binary stars with current instrumentation.
\end{abstract}

Key words: (stars:)binaries: general, instrumentation: interferometers

\section{INTRODUCTION}

The mass and radii of stars are nowadays most often determined from their asteroseismic frequencies, using scaling relations (e.g., Kjeldsen \& Bedding 1995) that relate stellar parameters to the solar values, or by comparing the frequencies with stellar-structure models (e.g., Rodrigues et al. 2017). As the asteroseismic methods are indirect, it is desirable to compare them, wherever feasible, with dynamical mass measurements and geometrical radius measurements, which directs attention to binary stars.

It has been known since Russell (1928) that combining astrometric and photometric observations of a binary stellar system yields the masses of both stars, as well as the three-dimensional orbit and the distance to the system. This method has long been used to measure the masses of stars in resolved binaries (e.g. Stephenson \& Sanwal 1969) and extended to binaries resolved by interferometry (e.g. Docobo et al. 2018). A further measurement of the radii of both stars would provide two mass-radius points.

Herbison-Evans et al. (1971) already succeeded in one mass-radius measurement. They observed the binary $\alpha$ Vir (Spica) using optical intensity interferometry at mas-scale resolution, as well as spectroscopy. Combining these observations they inferred the distance, the masses of both stars and the radius of the brighter star, as well as all the orbital parameters (see their Table III). Only the radius of the

\footnotetext{
* E-mail: niturai20129617@iisertvm.ac.in
}

second star was lacking. Unfortunately the progress of intensity interferometry stopped soon after, as the interest of the comunity shifted to other kinds of interferometry, and an interferometric measurement of the radius of the second star still remains to be done. However, a renewed interest in intensity interferometry and new observations resolving single stars (e.g., Acciari et al. 2020; Rivet et al. 2020; Abeysekara et al. 2020; Zampieri et al. 2021) suggest that it will soon become possible to use this technique to do mass-radius determinations in binary stars. The great advantage of intensity interferometry is that it does not require sub-wavelength precision on the mirrors or other optical components, and hence can be extended to very long interferometric baselines. Baselines of $>1 \mathrm{~km}$, corresponding to resolutions of $<1$ mas are planned (Dravins et al. 2013) and still longer baselines have been advocated (Baumgartner et al. 2020).

Intensity interferometry measures the squared visibility, or equivalently the $2 \mathrm{D}$ angular power spectrum of the source brightness, and from the observable it is possible to reconstruct the image of two stars (Dravins et al. 2013). In this work we adopt a different strategy, which is to take a parameterised model for two limb-darkened stars and fit their radii and separation to the interferometric observable.

This work discusses parameter estimation using simulated data for a binary. A Spica-like system is taken as an example, but no special properties of Spica are assumed. We consider two methods to recover the system parameters from the squared visibility. The first involves comparing the observable at two (or more) wavelengths to separate the visibility contributions of the two stars. Essential for this is that the two stars 
have different effective temperatures, and hence contribute differently at different wavelengths. The second method involves direct fitting of the squared visibility to a two-star model with parameters for the stellar radii, the projected distance, and limb darkening coefficient.

This paper is organized as follows. In Section 2, we describe the methods of separation in visibilities and direct fitting to estimate parameters, assuming a uniform-brightness model of the star. However, in reality, stars do not follow a uniform model of brightness. So in Section 3 we describe a limb darkening model of the stars. This is followed in Section 4 by simulations of intensity-correlation data and the recovery of the parameters of interest using a Markov chain Monte Carlo (MCMC) technique. The results our analysis are described in Section 5. Finally we conclude in Section 6 .

\section{METHODOLOGY}

An amplitude interferometer measures the modulus and phase of the Fourier transform of the brightness distribution of an incoherent source. Hence the response of the amplitude interferometer, if sufficiently well sampled, can be inverted to obtain the original image of the source. Techniques for doing so are well known, especially in radio-astronomy (see e.g., Thompson et al. 2017, section 10.4). The processing of an image obtained in this way, is heavily dependent on the ability of the measuring device to accurately track the phase of the light signal coming from sources. The latter is not required in intensity interferometry, which measures only the modulus of the Fourier transform of a source's brightness distribution. Because of the lack of phase information, reconstruction of a source having a complex structure requires information to be added, as a model or other prior. In the case where the source's shape is known, a precise size estimate can be extracted in a model-dependent manner.

\subsection{Correlated Signal}

We consider first a binary source comprising two stars $A$ and $B$ of uniform brightness with angular radii $\Theta_{A}$ and $\Theta_{B}$, and effective temperature $T_{A}$ and $T_{B}$ respectively, at angular distance $\Theta_{d}$ from each other. The total flux of photons coming from the binary source is the weighted sum of the photon flux coming from the individual stars

$$
\Phi=I_{A}+I_{B}=\left|S_{A}(\Omega)\right|^{2} \pi \Theta_{A}^{2}+\left|S_{B}(\Omega)\right|^{2} \pi \Theta_{B}^{2}
$$

where $I_{A}=\left|S_{A}(\Omega)\right|^{2}$ and $I_{B}=\left|S_{B}(\Omega)\right|^{2}$ are the flux of photons from stars $A$ and $B$ respectively.

For a star of uniform brightness, visibility function at a point $(u, v)$ on the interferometric plane has the well-known form

$$
V_{A}(u, v) \propto \frac{J_{1}\left(\rho \Theta_{A}\right)}{\left(\rho \Theta_{A}\right)}
$$

and analogously for $V_{B}(u, v)$, where

$$
\rho=\sqrt{u^{2}+v^{2}}
$$

For both stars together the corresponding visibility function is

$$
V(u, v)=\frac{I_{A} V_{A}(u, v)+I_{B} V_{B}(u, v) \exp \left(i \rho \Theta_{d} \cos \psi\right)}{I_{A}+I_{B}}
$$

where the $u$ axis of the baseline is oriented along the line connecting the sources. The square of the modulus of the visibility function

$|V(u, v)|^{2}=\frac{\left[\begin{array}{c}I_{A}^{2} V_{A}^{2}(u, v)+I_{B}^{2} V_{B}^{2}(u, v) \\ +2 I_{A} I_{B} V_{A}(u, v) V_{B}(u, v) \cos \left(\rho \Theta_{d} \cos \psi\right)\end{array}\right]}{\left[I_{A}+I_{B}\right]^{2}}$

is the main observable in intensity interferometry. By construction $V(0,0)=1$. In practice, however, the measured quantity is

$$
g(u, v)=\frac{\Delta \tau}{\Delta t}|V(u, v)|^{2}
$$

where $\Delta \tau$ is the coherence time or equivalently the reciprocal of the frequency bandwidth (Mandel \& Wolf 1965) while $\Delta t$ is the time resolution.

\subsection{Methods to fit parameters}

The discussion of this paper is based on two possible strategies, which we now describe, to estimate the parameters of the stars.

\subsubsection{Separation in visibilities}

In this method, we consider measurements at two different wavelengths. Provided the stars have different effective temperatures, the squared visibility will be measured with two different values of the flux ratio $f=I_{B} / I_{A}$. Then there is a possibility to estimate both radii of the system.

Along the $v$ axis, i.e., $u=0$ the visibility (4) will be real. We may then take the square root of the squared visibility (5) to obtain

$$
V_{1}(0, v)=\frac{V_{A}(0, v)+f_{1} V_{B}(0, v)}{1+f_{1}}
$$

and

$$
V_{2}(0, v)=\frac{V_{A}(0, v)+f_{2} V_{B}(0, v)}{1+f_{2}}
$$

Assuming the sign of $V_{1}(0, v)$ and $V_{2}(0, v)$ can be inferred, the visibility contributions of source $A$ and source $B$ in terms of $V_{1}(0, v)$ and $V_{2}(0, v)$ are

$$
V_{A}(0, v)=\frac{\left[\begin{array}{c}
V_{1}(0, v) f_{1} f_{2}-V_{2}(0, v) f_{1} f_{2} \\
+V_{1}(0, v) f_{2}-V_{2}(0, v) f_{1}
\end{array}\right]}{f_{2}-f_{1}}
$$

and

$$
V_{B}(0, v)=\frac{\left[\begin{array}{c}
f_{1} V_{1}(0, v)-f_{2} V_{2}(0, v) \\
+V_{1}(0, v)-V_{2}(0, v)
\end{array}\right]}{f_{1}-f_{2}}
$$

The expressions (9) and (10) can now be used to fit parameters, specifically the radii of both stars. But using this method, the distance between stars can not estimated as the above equation are independent of $\Theta_{d}$. 


\subsubsection{Direct fitting}

The second method we consider is directly fitting the squared visibility (5) to infer the parameters. Since $|V(u, v)|^{2}$ depends explicitly on $\Theta_{d}$ as well as on $\Theta_{A}$ and $\Theta_{B}$, we can use this correlation to estimate the angular separation between the sources together with angular radii of the sources.

\subsection{Slope of the Signal}

In order to better understand the dependence of the squared visibility on each of the radii in different parts of the $u, v$ plane, we consider derivatives of the former (equ. 5) with respect to $\Theta_{A}$ and $\Theta_{B}$ (taking $I_{A}$ and $I_{B}$ as normalised constants) for $u=0$, which are as follows.

$$
\frac{\partial|V(0, v)|^{2}}{\partial \Theta_{A}}=-\frac{4 I_{A} V(0, v)}{\left(I_{A}+I_{B}\right)} \frac{J_{2}\left(v \Theta_{A}\right)}{\Theta_{A}}
$$

and

$$
\frac{\partial|V(0, v)|^{2}}{\partial \Theta_{B}}=-\frac{4 I_{B} V(0, v)}{\left(I_{A}+I_{B}\right)} \frac{J_{2}\left(v \Theta_{B}\right)}{\Theta_{B}}
$$

The first factor of eqns. (11) and (12) depends on both sources. However, the second factor depends on only the source with respect to which equ. (5) is differentiated. As we can see, the derivative is a product of Bessel functions $J_{1}$ and $J_{2}$, and can be positive or negative. So, there will be some regions in the $u, v$ plane where the derivative of the observable will be positive and some regions where it will be negative. Using these equations, we can remove the data from the baseline region that reduces the strength of the signal (i.e., the area of baselines from where signals with negative slope observed).

\section{IMPACT OF LIMB-DARKENING}

Limb darkening is the phenomenon that describes the brightness of a star as a function of its radius, observed relative to the maximum brightness at the center of the stellar disk. The disk of the star is dimmer towards the limbs than at the center. As first explained by Schwarzschild (1906) limb darkening occurs due to the gradient of temperatures through the layers of the star. Higher temperatures emit more photons at a given wavelength, and lower temperatures emit fewer. The photons from a star are emitted from one optical depth within the surface of the star. Because of the star's geometry, one optical depth at the center of the stellar disk penetrates through to a deeper layer of the star than one optical depth near the limb; therefore, we see a hotter region, and thus middle of the stellar disk appears brighter.

Limb darkening is typically treated as a simple parameterised function of the angle between a line normal to the stellar surface and the observer's line of sight, which makes fitting the stellar intensity profile much simpler and reduces the number of free parameters. The most common parametrizations are linear and quadratic relations (Kopal 1950), but other suggested relations include a four-parameter relation, a square-root relation as well as exponential and logarithmic relations (Milne 1921; Diaz-Cordoves \& Gimenez 1992; Klinglesmith \& Sobieski 1970; Claret \& Hauschildt 2003; Sing et al. 2009; Claret 2000). Following the work described in
Hanbury Brown et al. (1974) we have used a limb darkening model of the form

$$
\left|\frac{S(\Omega, \mu)}{S(\Omega, 1)}\right|^{2}=1-l(1-\mu)-m(1-\mu)^{2}-n(1-\mu)^{3}
$$

where $l, m$ and $n$ are limb coefficient depending on the surface temperature and frequency, and $\mu$ is the cosine of the angle $\theta$ between the normal to the surface at that point and the line of sight from the star to the observer. In terms of the radius $R$ of the star, the projected radial position $R^{\prime}$ of the observed point, and the angle $\theta$ is expressed as

$$
\mu=\cos \theta=\sqrt{1-\frac{R^{\prime 2}}{R^{2}}}
$$

Given the limb darkening model under consideration and the under the assumptions that the values of the limb-darkening coefficients are frequency independent and same for both the stars, the total flux of binary source is given by

$$
\Phi=C\left(I_{A}+I_{B}\right)
$$

where the constant $C$ in terms of limb coefficients is given by,

$$
C=\left(1-\frac{l}{3}-\frac{m}{6}-\frac{n}{10}\right)
$$

and the visibility functions of star $A$ and $B$ are given by

$$
\begin{aligned}
& V_{A}(u, v)=\sum_{i} a_{i} \frac{J_{i}\left(\rho \Theta_{A}\right)}{\left(\rho \Theta_{A}\right)^{i}} \\
& V_{B}(u, v)=\sum_{i} a_{i} \frac{J_{i}\left(\rho \Theta_{B}\right)}{\left(\rho \Theta_{B}\right)^{i}}
\end{aligned}
$$

For $i=1,3 / 2,2,5 / 2$ and the value of $a_{i}$ for each $i$ are

$$
\begin{aligned}
& a_{1}=1-l-m-n \\
& a_{3 / 2}=\sqrt{\frac{\pi}{2}}(l+2 m+3 n) \\
& a_{2}=-2(m+3 n) \\
& a_{5 / 2}=3 \sqrt{\frac{\pi}{2}} n
\end{aligned}
$$

In the absence of star $B$, the result will be according to Hanbury Brown et al. (1974).

Eqns. (17) and (18) are the generalized form of visibilities of sources $A$ and $B$, according to the coefficient parameters $l, m, n$ the limb darkening model of star assumed here.

\section{SIMULATIONS AND PARAMETER FITTING}

We now proceed to simulate intensity-correlation data and recover the parameters using a Markov chain Monte Carlo (MCMC) algorithm.

\subsection{Signal to noise}

We may write the signal as

$$
g(u, v, \omega)=\frac{\Delta \tau}{\Delta t}|V(u, v, \omega)|^{2}
$$

which is equ. (6) slightly rewritten with $\omega$ standing for all the parameters of the system (radii, distance, and limb-darkening coefficients). For the signal at the $i$-th baseline, we write

$$
g_{i}\left(u_{i}, v_{i}, \omega\right)=\frac{\Delta \tau}{\Delta t}\left|V\left(u_{i}, v_{i}, \omega\right)\right|^{2}
$$


and the data collected at that baseline will be

$$
d_{i}=g_{i}+\sigma_{i} \mathcal{N}_{i}
$$

where $\sigma_{i}$ is the noise level and $\mathcal{N}_{i}$ is a Gaussian random number. ${ }^{1}$ The signal-to-noise at the $i$-th baseline will be

$$
\mathrm{SNR}_{i}=\frac{d_{i}}{\sigma_{i}}
$$

while

$$
\mathrm{SNR}=\left(\sum_{i} \mathrm{SNR}_{i}^{2}\right)^{1 / 2}
$$

will be the total signal-to-noise ratio for a full simulation.

For the visibility-separation method, the relation between $\mathrm{SNR}_{i}$ and $\sigma_{i}$ is more complicated. First, the observable squared visibility has to be converted to individual visibilities using eqns. (7) and (8). Correspondingly, the $\mathrm{SNR}_{|V|^{2}}$ (say) relating to the observable, has to be converted to an effective

$$
\mathrm{SNR}_{V}=2 \mathrm{SNR}_{|V|^{2}}
$$

using the error propagation formula. Further using the latter formula we have

$$
\sigma_{V_{A}}^{2}=\left(\frac{\partial V_{A}}{\partial V_{1}}\right)^{2} \sigma_{V_{1}}^{2}+\left(\frac{\partial V_{A}}{\partial V_{2}}\right)^{2} \sigma_{V_{2}}^{2}
$$

and similarly $\sigma_{V_{B}}^{2}$. The values of $\sigma_{V_{A}, i}$ and $\sigma_{V_{B}, i}$ are required for parameter-fitting.

To relate the SNR in a simulation to the expected SNR in an observing run, we recall that for one baseline

$$
\mathrm{SNR}_{i} \approx\left(A_{1} A_{2}\right)^{1 / 2} \Phi\left|V\left(u_{i}, v_{i}\right)\right|^{2}\left(t_{i}^{\text {obs }} / \Delta t\right)^{1 / 2}
$$

where $A_{1}$ and $A_{2}$ are the effective collecting areas,

$$
\Phi \approx \frac{\lambda}{1 \mu \mathrm{m}} \times 10^{-4-\mathrm{AB} / 2.5} \text { photons } \mathrm{m}^{-2} \mathrm{~s}^{-1} \mathrm{~Hz}^{-1}
$$

is the spectral photon density per polarisation channel, and $t_{i}^{\text {obs }}$ is the observing time at that baseline. The full SNR of the observing run is then obtained using equ. (24) as before.

A counterintuitive fact here is that while the observable $g$ depends on the coherence time $\Delta \tau$, the SNR is independent of $\Delta \tau$. This was first noted by Hanbury Brown \& Twiss (1957) and can be understood as follows. Suppose we change the bandwidth by a factor of $k$, while keeping the detector timeresolution $\Delta t$ fixed. This is equivalent to changing the $\Delta \tau$ by $1 / k$. There will be $k$ times as many photons in each detector per $\Delta t$, and hence $k$ times as many intensity-interferometric correlations - not $k^{2}$ times, because photons in different slices of the band do not interfere. However, the $k$ times as many photons will have $k^{2}$ times as many chance coincidences, implying $k$ times as much shot noise. As a result both signal and noise will change by the same factor $k$. We remark that all of this is valid only if the coherence time $\Delta \tau$ is much smaller than the instrumental time resolution $\Delta t$, which is a safe assumption for any foreseeable instrumentation. Furthermore, for intensity interferometry with three-photon or higher-order correlations the SNR does depend on $\Delta \tau$ (see e.g., Malvimat et al. 2014).

1 The noise level depends on the observing time at each baseline, and in the simulations we assume all the $\sigma_{i}$ to the same for all baselines, though this is not essential.

\subsection{Parameter fitting}

For a given data set the likelihood in the usual Bayesian formulation will be

$$
\ln P\left(\left\{d_{i}\right\} \mid \alpha, \omega\right)=-\frac{1}{2} \sum_{i} \sigma_{i}^{-2}\left(d_{i}-\alpha\left|V\left(u_{i}, v_{i}, \omega\right)\right|^{2}\right)^{2}
$$

where $\alpha=\Delta \tau / \Delta t$ is an additional parameter, which will not be accurately known in advance. We can marginalise out the nuisance parameter $\alpha$ to avoid having to fit it, as follows. Assuming a flat prior for $\alpha$ we have

$$
P\left(\left\{d_{i}\right\} \mid \omega\right)=\int P\left(\left\{d_{i}\right\} \mid \alpha, \omega\right) d \alpha
$$

We now define two scalar products: first of the data with the model

$$
G=\sum_{i} \sigma_{i}^{-2} d_{i}\left|V\left(u_{i}, v_{i}, \omega\right)\right|^{2}
$$

and, the scalar product of model with itself

$$
W=\sum_{i} \sigma_{i}^{-2}\left|V\left(u_{i}, v_{i}, \omega\right)\right|^{4}
$$

The integral (29) can now be expressed as

$$
\ln P\left(\left\{d_{i}\right\} \mid \omega\right)=\frac{G^{2}}{2 W}-\frac{1}{2} \ln W
$$

Eqns. (31-33) are convenient for input to MCMC. An MCMC implementation, such as the well-known emcee code (Foreman-Mackey et al. 2013) which we used, generates a sample of points $\omega$ in parameter space sampled according to some given function of $\omega$. Sampling is desired according to $P\left(\left\{d_{i}\right\} \mid \omega\right)$ times the prior probability of $\omega$, the latter representing the allowed ranges of the various parameters. The resulting sample of $\omega$ points cannot in general be visualised directly, and hence projections to one- and two-parameter subspaces are conventionally used, as in Fig. 5.

\section{RESULTS}

In this section we first describe the signal of our interest i.e., visibility and squared visibility. This is followed by the estimation of parameters in two ways: first by separation of visibilities, and second the direct fitting of the squared visibility.

The parameter values used for the simulations are $\Theta_{A}=$ 2.20, $\Theta_{B}=1.10$ nanoradians for the angular radii and $\Theta_{d}=$ 8.30 nanoradians for the angular separation. ${ }^{2}$ These values correspond roughly to the Spica system Herbison-Evans et al. (1971); Tkachenko et al. (2016). Simulated observations at $\nu=5 \times 10^{14} \mathrm{~Hz}$ and $\nu=7 \times 10^{14} \mathrm{~Hz}$ are considered. For brevity we refer to these as red and blue.

The ratio $\Delta \tau / \Delta t$ of coherence time to resolution time is taken to be 0.11 . The latter may be much lower in practice. For example, (Zampieri et al. 2021) observed with $(\lambda, \Delta \lambda)$ values of $(656.7 \mathrm{~nm}, 3 \mathrm{~nm})$ and $(510.5 \mathrm{~nm}, 0.3 \mathrm{~nm})$ amounting to $\Delta \tau=\lambda^{2} /(c \Delta \lambda)$ of about $0.5 \mathrm{ps}$ and $3 \mathrm{ps}$ respectively, whereas $\Delta t \approx 400 \mathrm{ps}$. However, as explained in the previous section, the SNR does not depend on $\Delta \tau$, and in the simulations $\Delta \tau / \Delta t$ is simply an arbitrary normalisation which gets marginalised out as a nuisance parameter.

\footnotetext{
2 Recall that a mas is about 5 nanoradians.
} 


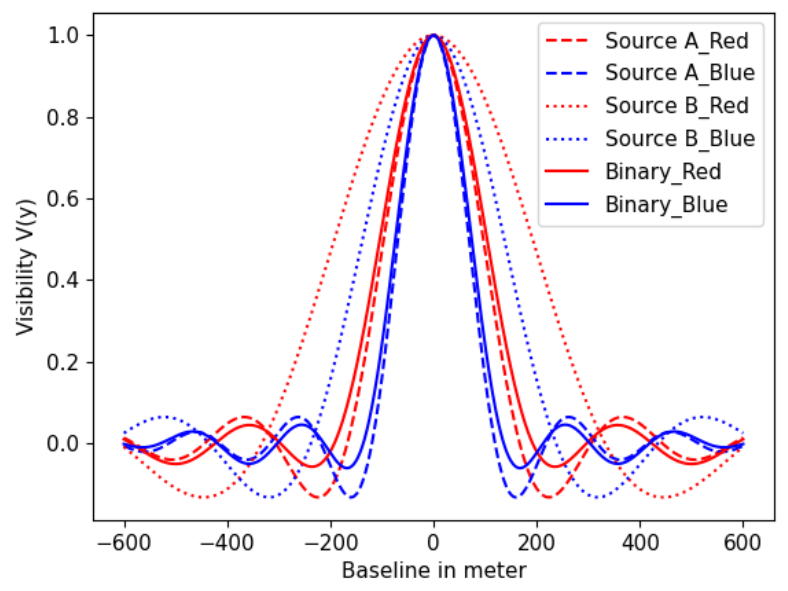

(a) Visibility along the baseline

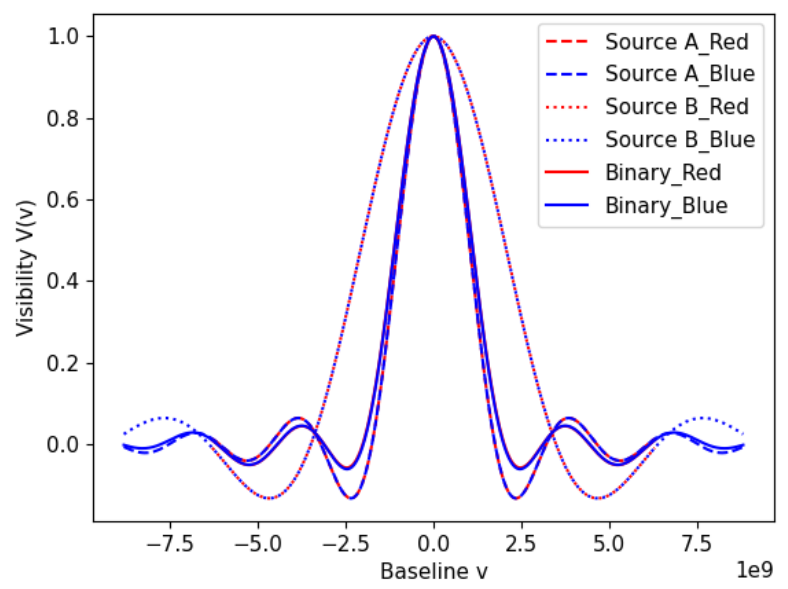

(c) Visibility along unit-less baseline

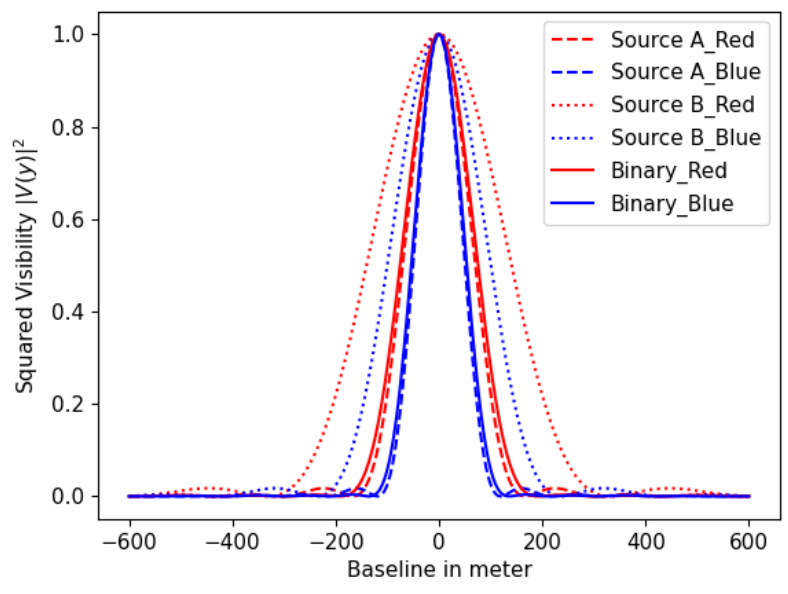

(b) Squared visibility along baseline

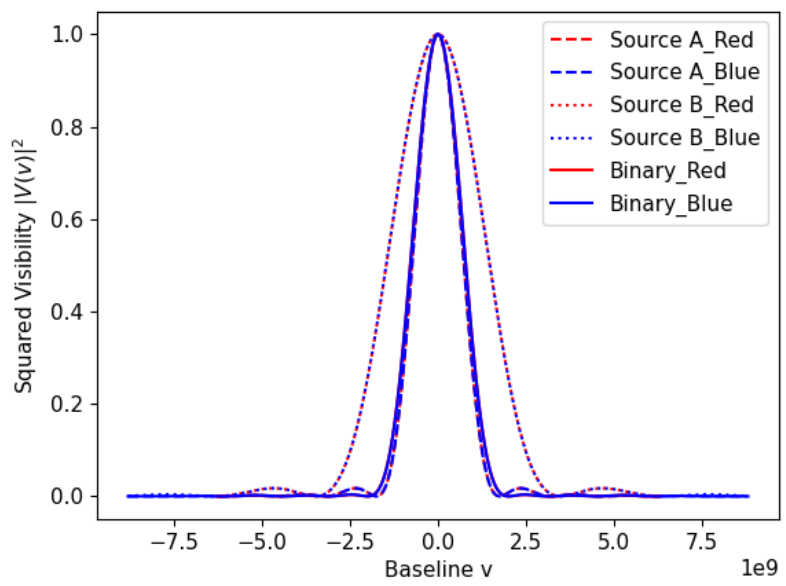

(d) Squared visibility along unit-less baseline

Figure 1. Analytical plot of the signal at two different wavelengths, for baselines perpendicular to the line between the stars. The two left panels show the visibility, while the two right panels show the squared visibility. For the two upper panels the baseline is in metres, while the two lower panels use the dimensionless baseline $v$. According to eqns. (4) and (5) the signal is always unity at zero baseline.

\subsection{Signal}

Fig. 1 shows the main signal for baselines perpendicular to the orientation of the binary. As expected, the signal attains its maximum value for zero baseline, and for a single star the larger the source size the narrower is the visibility pattern. However, the visibility of the total system is weighted towards the brighter source. Source A dominates the brightness both in blue and red, but is slightly less dominant in red; this difference is, however, not discernible from the figure. For certain wavelength (most noticibly around $175 \mathrm{~m}$ at blue wavelength) the visibility $V(0, v)$ is found to be less for the binary system than those for either of the sources under consideration. This is due to the fact that the visibilities for sources have opposite sign and are interfering destructively.

\subsection{Measuring Parameters}

For visibility separation, the values of the parameters $f_{1}$ and $f_{2}$ were assumed to be 0.10 and 0.11 respectively. The separation has been done for different values of SNR as shown in Fig. 2. The resulting estimates of the angular radii are shown in Fig. 3. Our results show that the minimum value of SNR for which this method works is around 300 .

For direct fitting of the squared visibility, we simulated the HBT correlation (Fig. 4a) along the baseline for $\cos \psi=$ $0,0.5,1$. It is not necessary to have the signal along straight lines in the $(u, v)$ plane, but this choice conveniently illustrates the oscillatory features in the correlation, which are due to the presence of a cosine factor in the expression of correlation equ. (5). The added noise is such that the total $\mathrm{SNR}=370$. Fig. 5 clearly shows good agreement between input and recovered values of the parameters. Although Fig. 5 shows no correlation between angular separation and angular radii of either star and, however, it shows positive correlation 


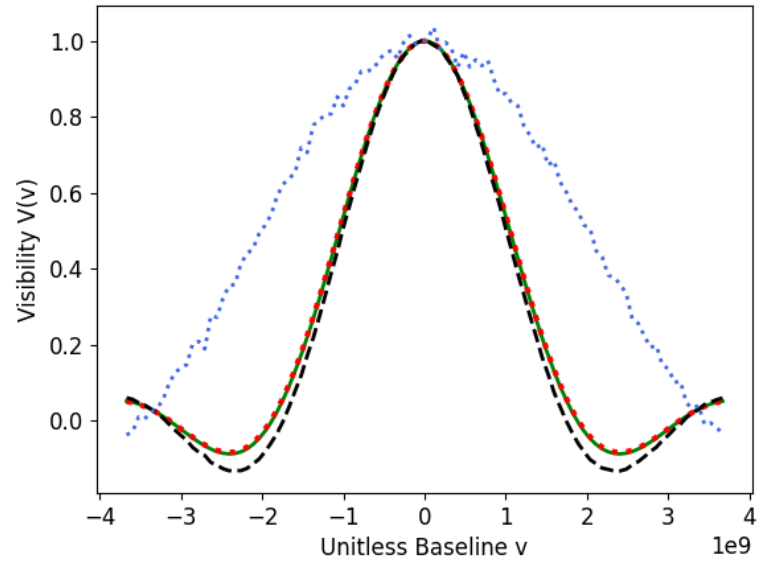

(a) $\mathrm{SNR}=32700$

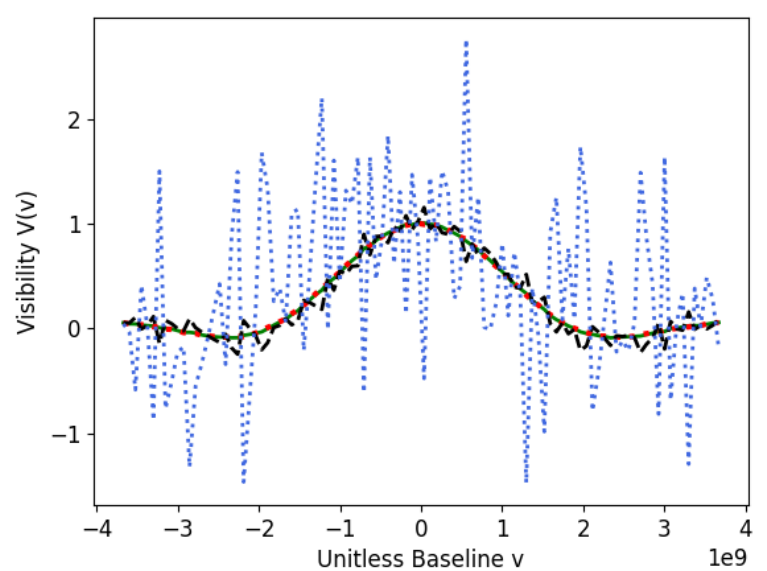

(b) $\mathrm{SNR}=650$

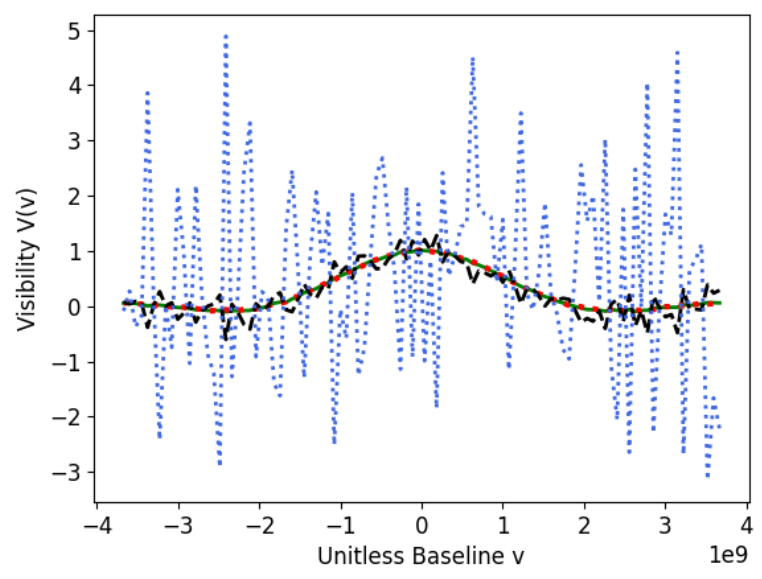

(c) $\mathrm{SNR}=320$

Figure 2. Visibility separation from simulated data illustrated at different SNR levels. The dotted red and plane green line show the combined visibility with $f_{1}=0.1$ and $f_{2}=0.11$ respectively (see eqns. 7 and 8). The baseline $v$ is perpendicular to the line between the two sources. The dashed black line and the dotted royalblue line show the inferred individual visibilities of source $A$ and source $B$ respectively.

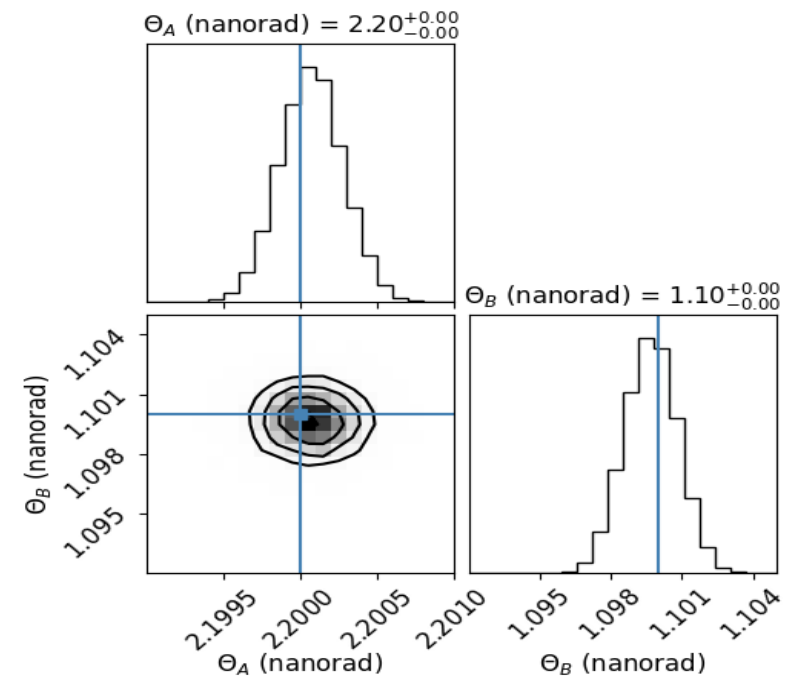

(a) Corner plot for $\mathrm{SNR}=32700$

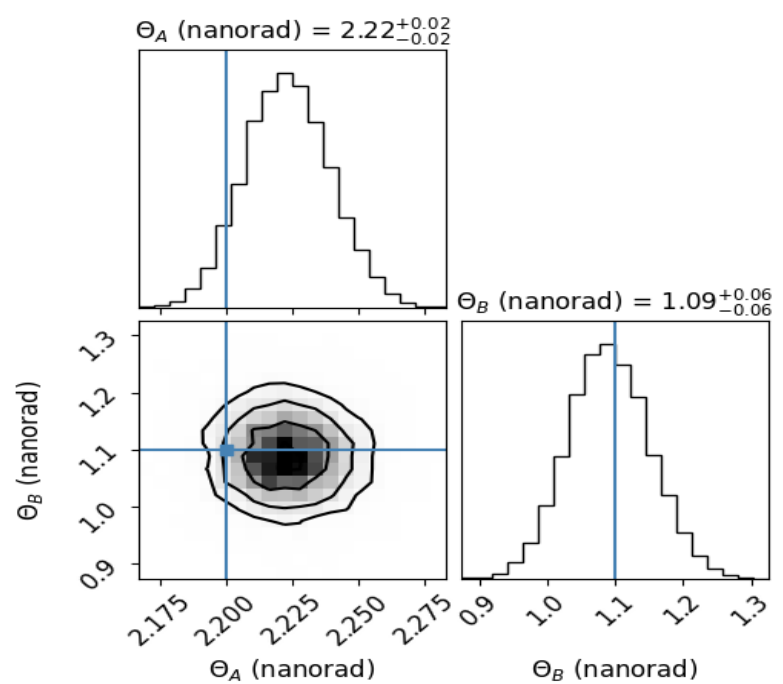

(b) Corner plot for $\mathrm{SNR}=650$

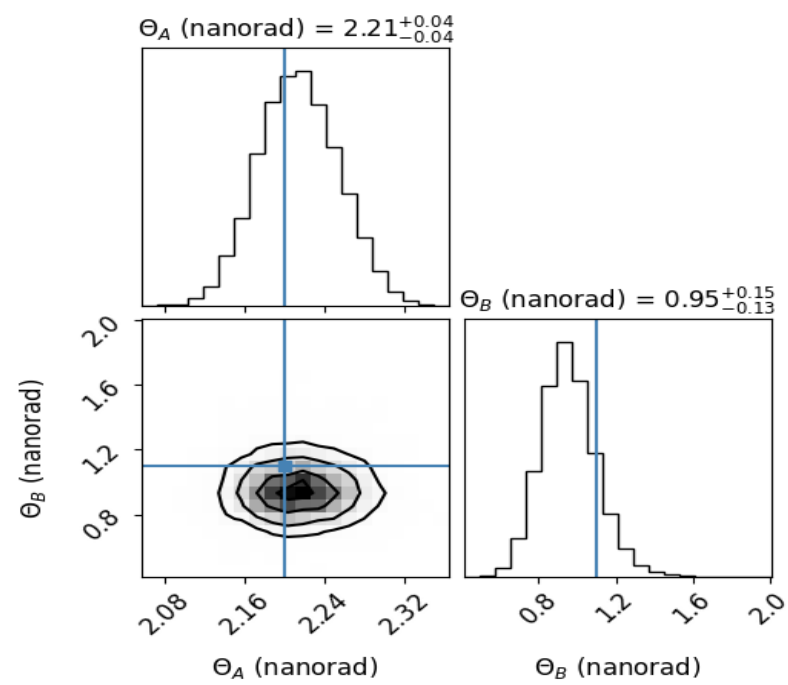

(c) Corner plot for $\mathrm{SNR}=320$

Figure 3. Estimation of the angular radii (in nanorad) of stars $A$ and $B$ using separated visibilities shown in Fig. 2. 


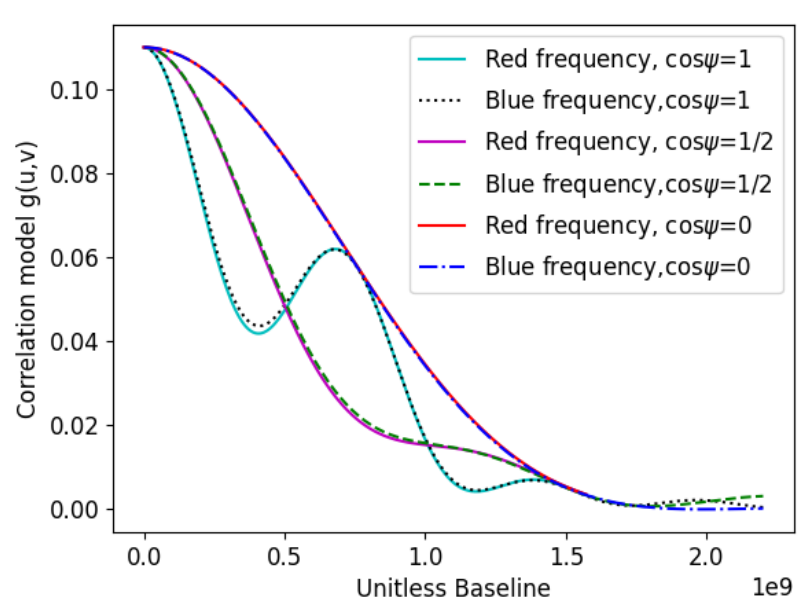

(a) Model data along baseline

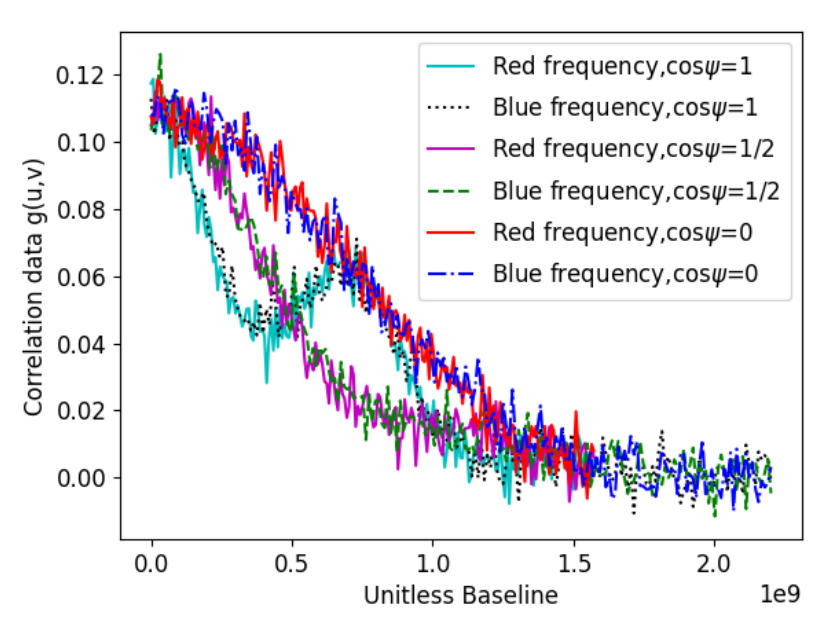

(b) Simulated data along baseline

Figure 4. Observable intensity correlation $g(u, v)$ at differently-oriented baselines and different observing wavelengths. The labelled $\cos \psi=u / \sqrt{u^{2}+v^{2}}$ where $u$ is along the line connecting the two stars. The left panel shows the model according to eqns. (5) and (6). The right panel shows the signal with added noise corresponding to a total SNR of 370 .

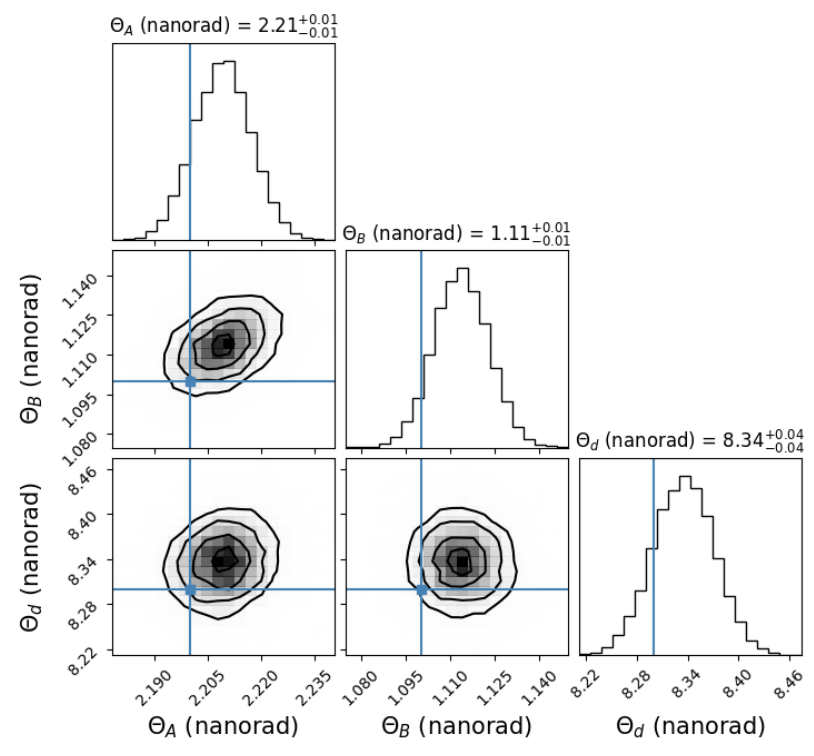

Figure 5. Estimation of the angular radii of the two stars and angular separation between them using the direct fitting method, from the simulated data shown in Fig. $4 \mathrm{~b}$. Note the positive correlation between angular radii of the two sources.

between the angular radii of the stars. In order to explain the origin of the correlation between the angular radii of the stars, we have plotted in Fig. 6 the change in squared visibility with angular radii of sources along baseline for the orientation of the sources in the direction perpendicular to the baseline. The sharp negative slope in Fig. 6 appears to be the cause of the correlation between estimated angular radii of the stars. In order to resolve this issue we tried removing from our analysis the unwanted portion of the baseline and followed the same methodology to estimate the parameters from the unmasked portion of the baseline, as shown in Fig. 7. The results in

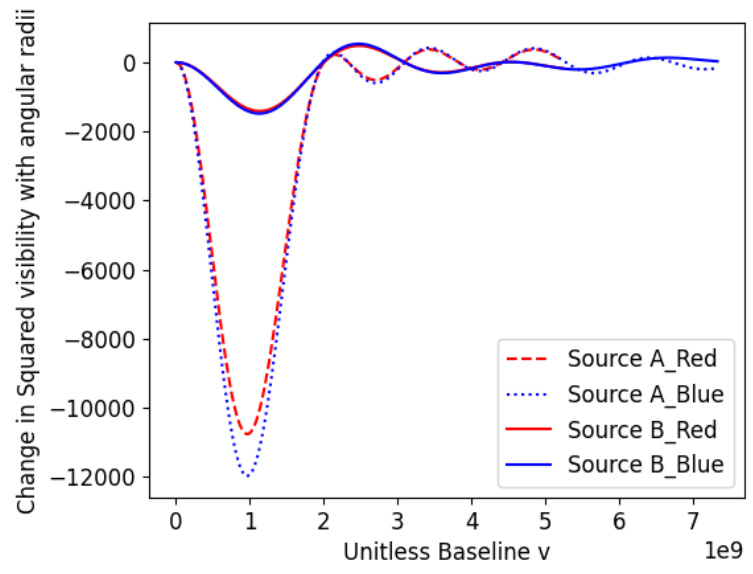

Figure 6. Derivatives of the squared visibility with respect to angular radii of the stars (eqns. (11) and (12)), shown along baselines perpendicular to the line connecting the stars. The regions with a sharp negative slope in the signal are considered for exclusion in the following simulations.

Fig. 8 show no correlation between the angular radii of the stars.

\subsection{Threshold Signal-To-Noise Ratio}

Estimation of parameters works requires a minimum value of the signal-to-noise ratio. Fig. 9 checks this threshold value of the SNR for estimation of angular radii of both sources and angular separation between them using a direct fitting with masking unwanted region. The minimum value of SNR is around 30. If the total SNR is much lower than in Fig. 9f, parameter estimation becomes poor, with the uncertainties becoming large and the credible regions excluding the true value. 


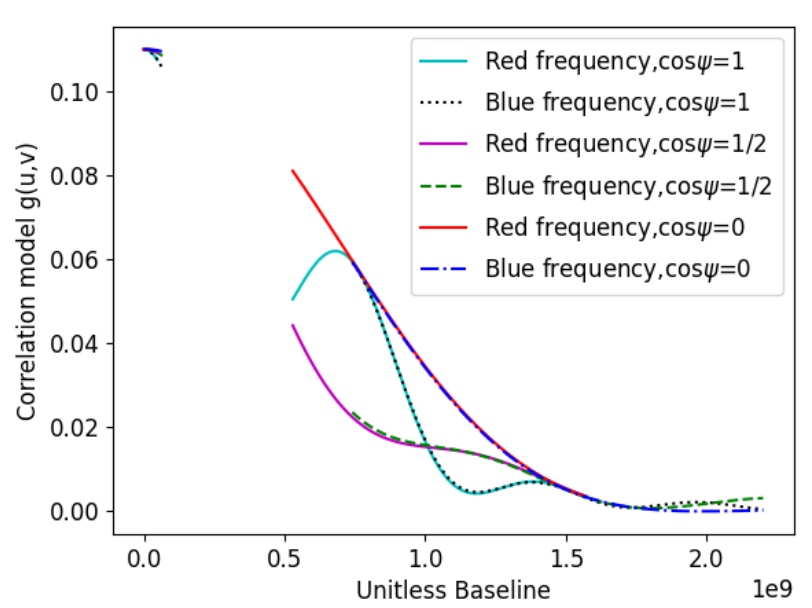

(a) Model data along baseline

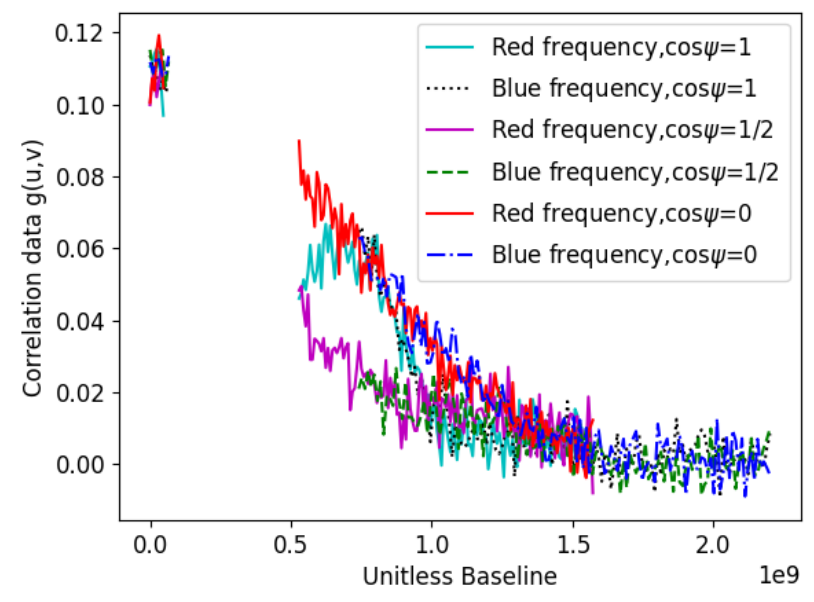

(b) Simulation data along baseline

Figure 7. Observable HBT correlation $g(u, v)$ after removing the region of baseline identified in Fig. 6. As Fig. 4 the left panel is for the model signal and the right panel adds noise. The total SNR here is 208 .

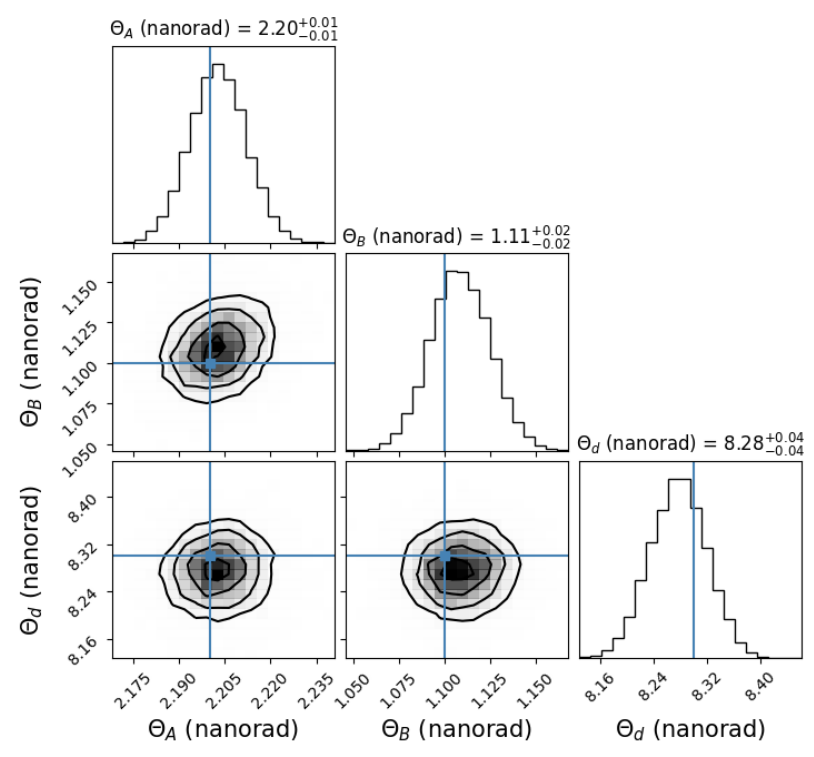

Figure 8. Estimation of the angular radii of the two stars and angular separation between them using the direct fitting method, from the simulated data shown in Fig. 7b. The difference from Fig. 5 is that some baselines regions have been excluded, with the result that the positive correlation between inferred angular radii of both sources has been mostly removed.

\subsection{Effect of limb-darkening}

In order to show the impact of limb-darkening on the squared visibility function of the stars visibilities for the orientation of the baseline corresponds to $\psi=0$. Fig. 10 shows that the impact of limb-darkening for three models: linear, quadratic and cubic. In all cases the difference due to limb darkening is small, and more prominent around the peaks. Investiging further we have found that the effect is relatively more noticeable for the baseline range $(0.5-0.8) \times 10^{9}$ and $(1.1-1.5) \times 10^{9}$. It is to be noted that for the baseline $1.845 \times 10^{9}$, the squared visibility is always more for the limb darkened model than the uniform model. The reverse is the situation for the baseline higher than $1.845 \times 10^{9}$.

Fig. 11a shows the measurement of the parameters of the stars with limb-darkening taken into account. The input values of the limb-darkening coefficients used to generate the plots are $l=0.6, m=0.2$, and $n=0.1$ and signal-to-noiseratio equal to 370 . For estimating the parameters, angular radii of both stars, and angular separation between them, limb coefficients are taken as constant. From Fig. 10 and Fig. 11 it is clear that the effect of limb-darkening of stars can be measurable and estimation of limb-coefficient is also possible. Fig. 11 shows the measurement of all the limb-darkening coefficients together with the parameters of the stars. For the linear model of the limb-darkening of stars, we have good recovery of the limb darkening coefficients $l$. On the other hand, for complex limb darkening models the recovery of the nonlinear limb-darkening coefficients $m$ and $n$ is not unsuccessful (Fig. 11c and Fig. 11d). However, in all cases, we have managed to recover both angular radii and angular separation between stars also linear limb-darkening coefficient with good accuracy.

\section{DISCUSSION}

Recent years have seen a resurgence of interest in intensity interferometry, and many science cases have been discussed in the literature (Kieda et al. 2019). In this paper we argue that radius measurements of binary stars are an interesting science case for intensity-interferometry observatories now in development. The basic idea was known during the earlier generation of intensity interferometry: Herbison-Evans et al. (1971) observed Spica using intensity interferometry; combining the interferometric observations with spectroscopy, they reconstructed the binary orbit and inferred the distance to the system, weighed both the stars, and measured the radius of the brighter star; the radius of the second star was the main parameter that could not be determined. It is plausible that the signal-to-noise needed to measure the radius of the 


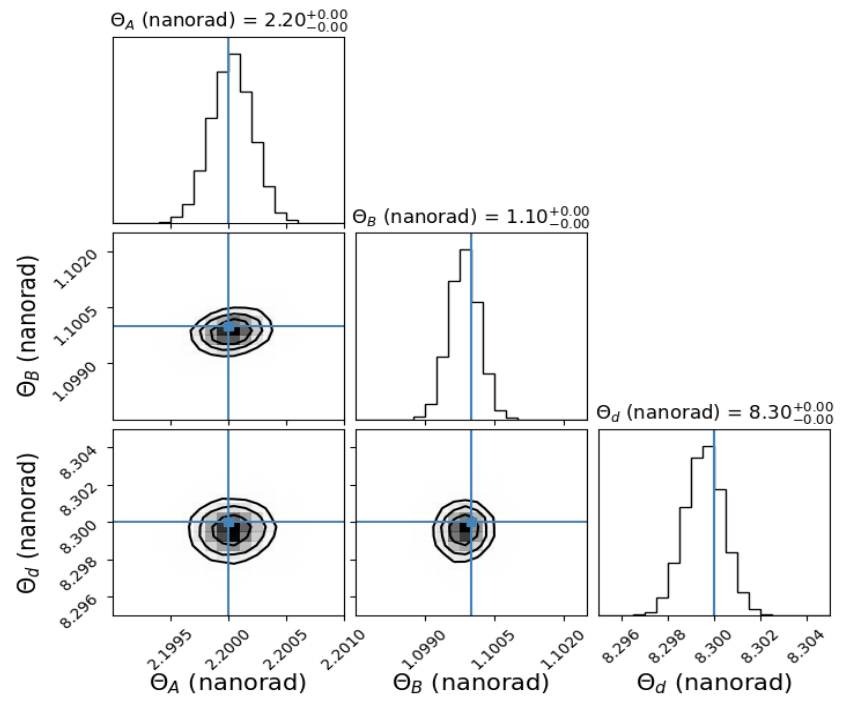

(a) $\mathrm{SNR}=10346$

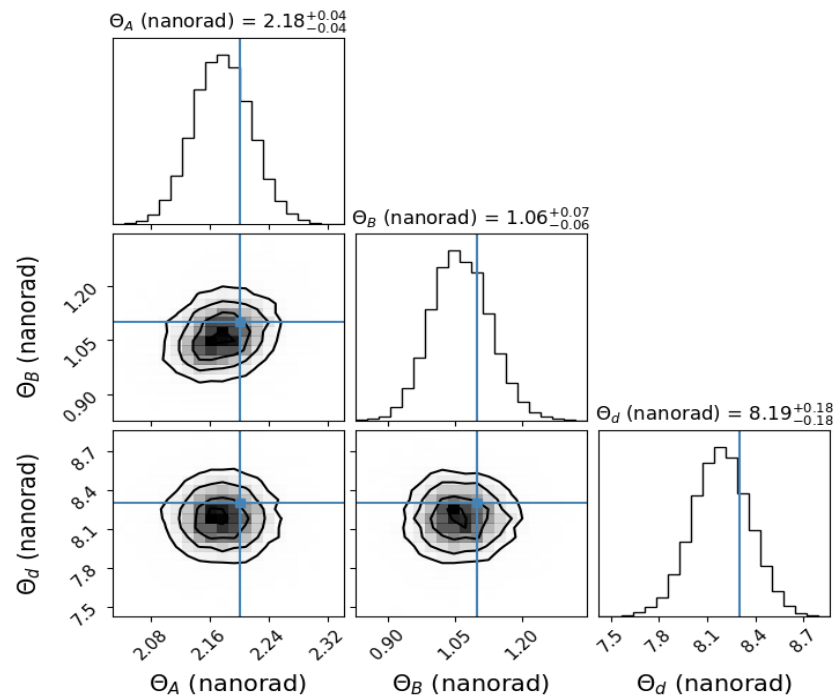

(c) $\mathrm{SNR}=55$

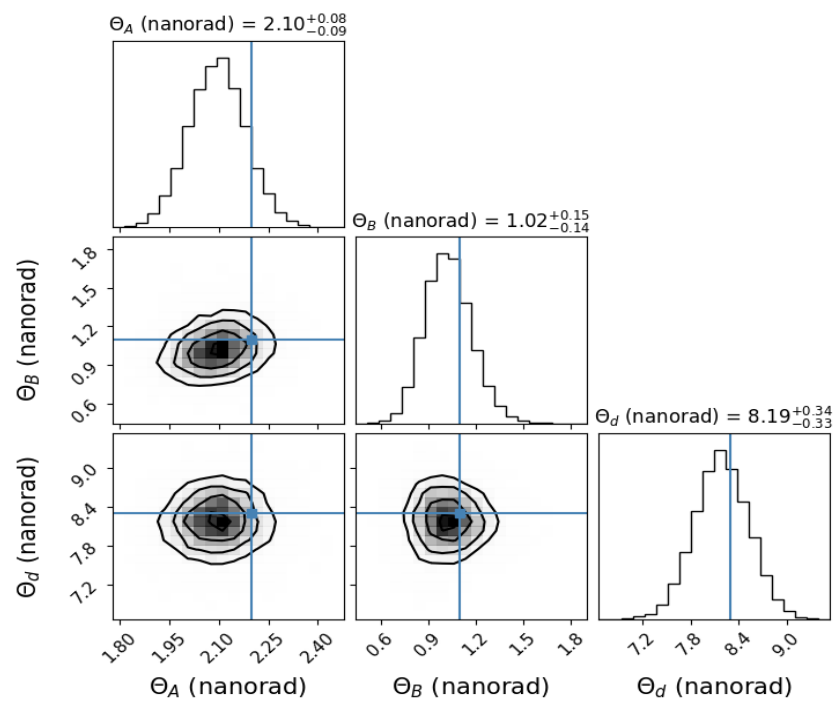

(e) $\mathrm{SNR}=35$

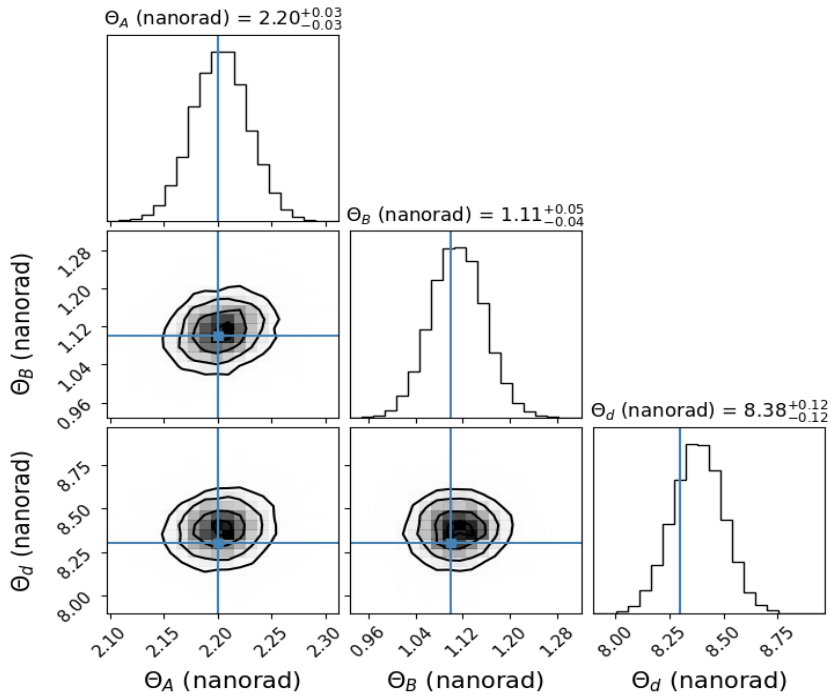

(b) $\mathrm{SNR}=79$

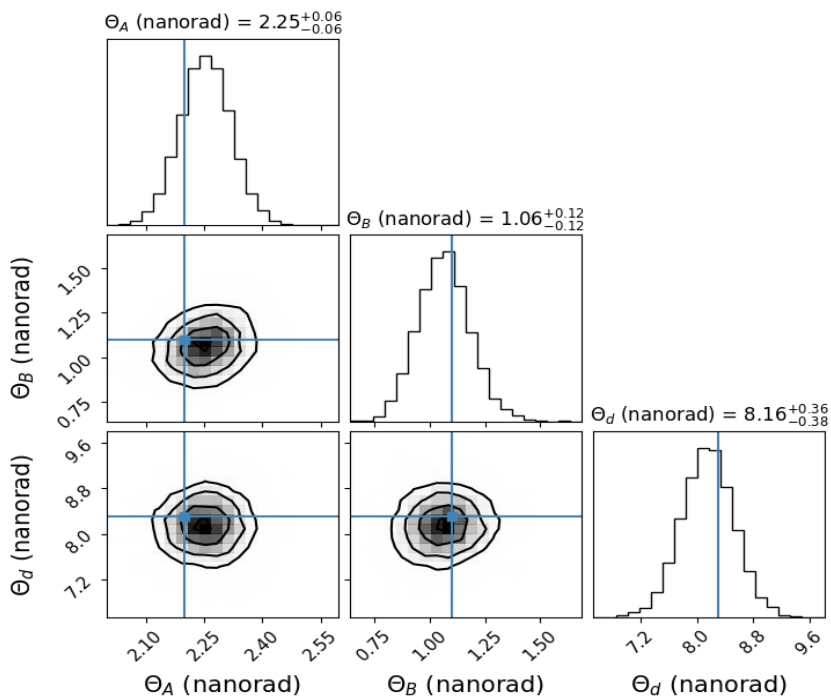

(d) $\mathrm{SNR}=41$

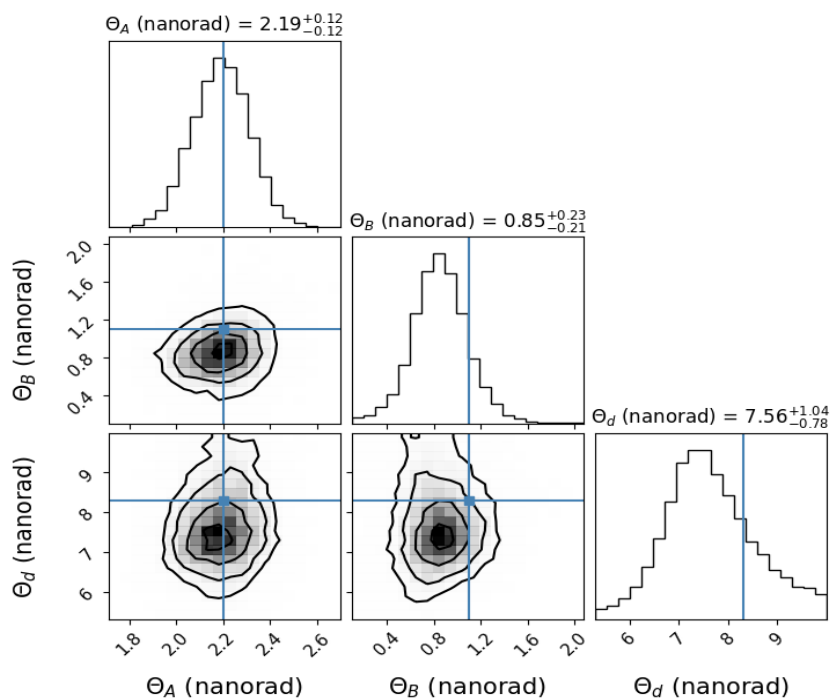

(f) $\mathrm{SNR}=32$

Figure 9. Estimations of the angular radii of both sources and the angular separation between them, using direct fitting at different total SNR. The threshold for estimating all three parameters is total SNR of about 30. 


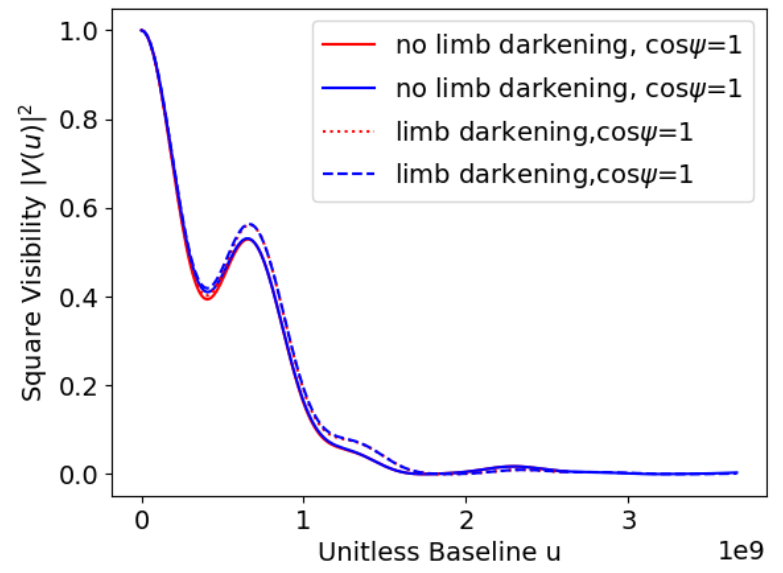

(a) limb coefficients $l=0.6, m=0.0, n=0.0$

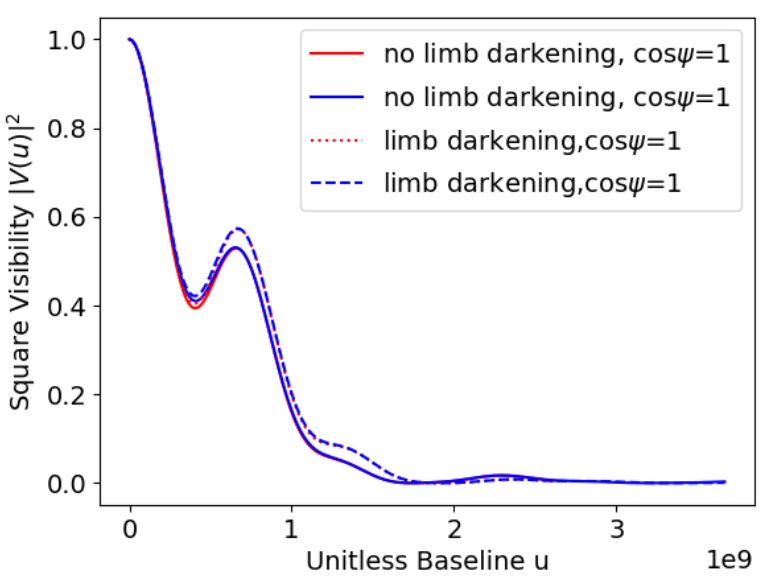

(b) limb coefficients $l=0.6, m=0.2, n=0.0$

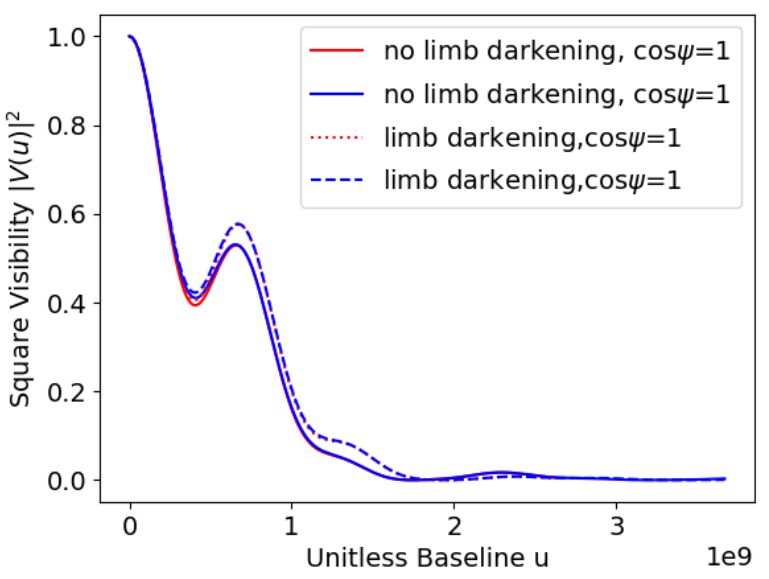

(c) limb coefficients $l=0.6, m=0.2, n=0.1$

Figure 10. Analytical plots of the squared visibility function, comparing a uniform-brightness model and limb-darkened models. The effect of limb darkening is measurable only near the peaks of the signal curve. The three panels show the differences between linear, quadratic and cubic limb-darkening, which are very small. second star was available even in 1971, but interferometric image reconstruction was still in its infancy. The interferometric pattern of a binary looks quite similar to a single star modulated with fringes (equ. (5)) and significant computing is needed to disentangle the contributions of two stars.

Nowadays, several image-reconstruction algorithms and codes are available (Berger et al. 2012) including developments specific to intensity interferometry (Nuñez \& Domiciano de Souza 2015). In particular, the reconstruction of an image of two stars from simulated data has been demonstrated (e.g., Figures 4-6 in Dravins et al. 2013). In this work, however, we suggest that rather than a general-purpose reconstruction of an image of two stars, parameter-fitting of the observable directed towards inferring the system parameters may be useful. Parameters that are not of interest but are unavoidable, such as the normalisation of the visibility, can be marginalised out. Markov chain Monte Carlo is well suited to this task, and efficient implementations are readily available. We used the emcee package.

In this paper we carry out a number of simulations, showing that with sufficient SNR, it is possible to extract both stellar radii, the angular separation between the stars, and linear limb-darkening. The SNR of the simulations can be related to source properties, telescope parameters, and observing time (eqns. 27 and 28) using well-known expressions for signal and noise in intensity interferometry. For example, suppose the spectral brightness times the effective area (eqns. 27 and 28) is $10^{-4} \mathrm{~s}^{-1} \mathrm{~Hz}^{-1}$. This will be the case for zero-magnitude source and an effective collecting around of about $1 \mathrm{~m}^{2}$, or a $5 \mathrm{mag}$ source and an effective collecting area of about $100 \mathrm{~m}^{2}$, and so on. With nanosec time resolution and an observing time of $10^{5} \mathrm{~s}(2-3$ nights $)$ the total SNR will be $\sim 1000$. The total observing time will of course be distributed among many different baselines. Thus, there could be 100 baselines obtained through pairs of light buckets and the Earth's rotation, each observed for $10^{3} \mathrm{~s}$ for SNR contribution of 100, added according to eqn. (24).

We consider two possible approaches to parameter-fitting for a binary-star system.

If the stars have different effective temperatures and hence differently-shaped spectra, and are observed at least two wavelengths, it is possible to separate the visibility contributions of each stars. There are several complications to the idea. The observable has to be square-rooted while taking into account possible sign changes. Moreover, the observations would need to be perpendicular to the line joining the stars, where the visibility is real. Then, visibility contributions have to be compared at the same values of dimensionless baseline at two different wavelengths, which will correspond to different lengths of the physical baseline. Finally, the visibility separation tends to amplify the noise. Nevertheless, the simulations indicate that measurement of both stellar radii is possible by this method.

Our second approach is multi-parameter fitting to the observed HBT correlation (squared visibility). Provided the parametric form being fitted is correct, one expects higher accuracy in the parameters. We consider a model with two discs in the sky with limb-darkening up to third order, at some separation from each other. Rough prior estimates on the radii of the two discs and their separation are assumed. A limb-darkening model with linear, quadratic, and cubic terms is used. If the limb-darkening parameters are assumed known, 


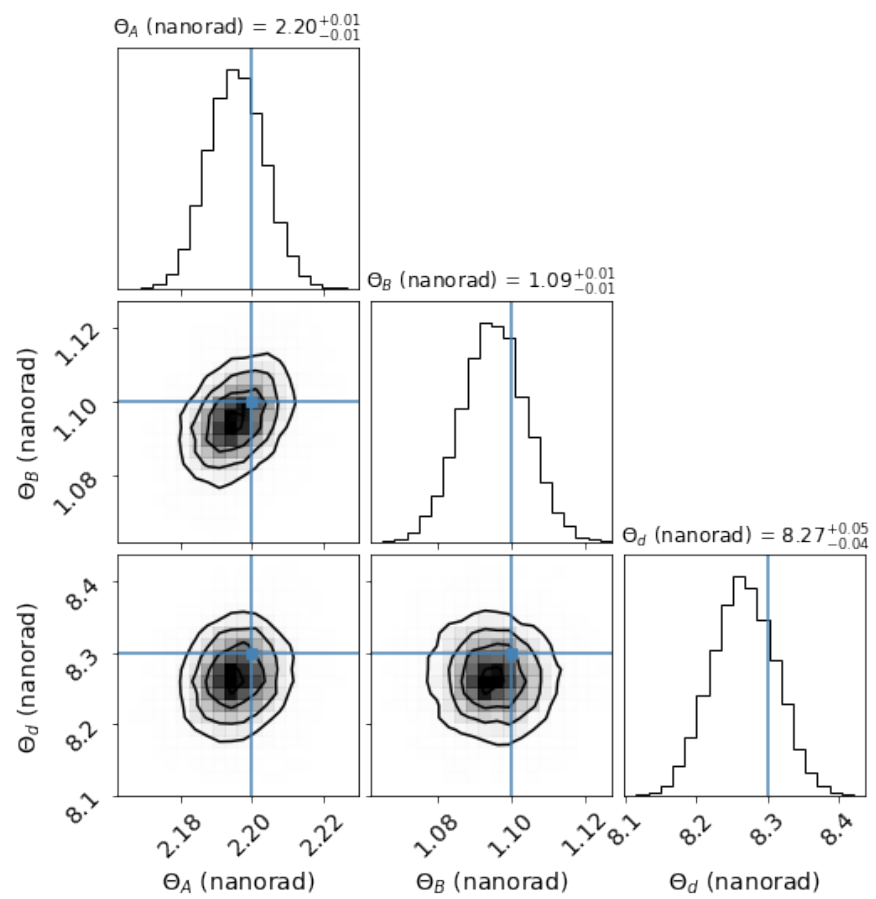

(a) $\mathrm{SNR}=370$

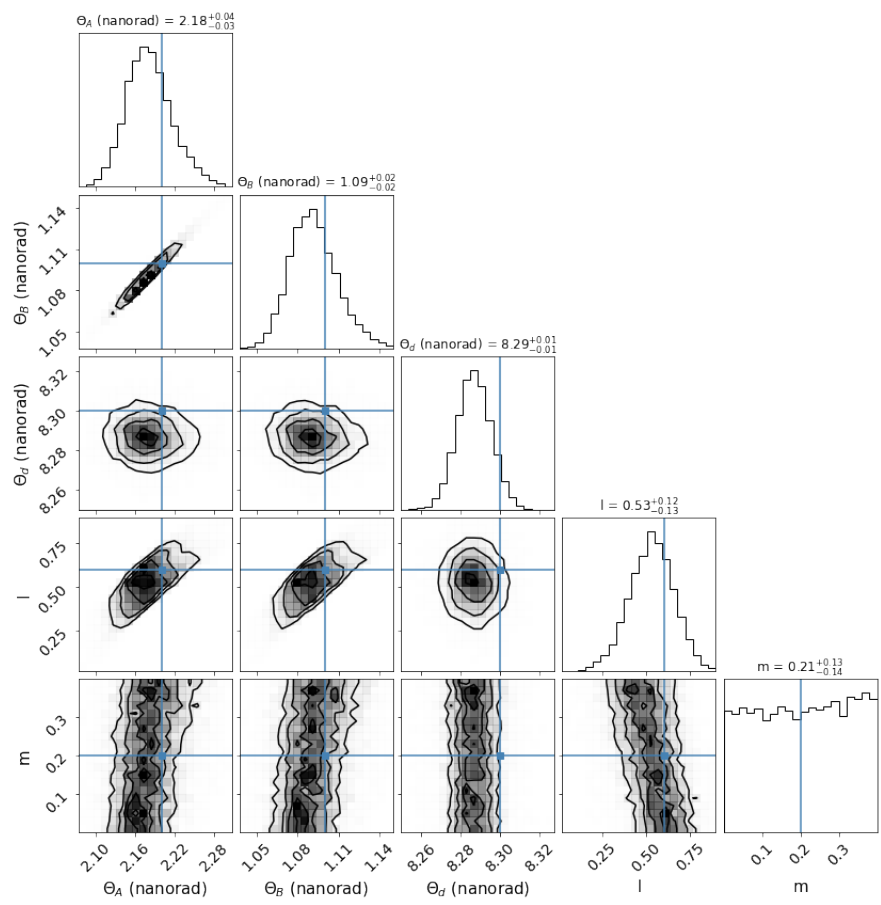

(c) $\mathrm{SNR}=1850$

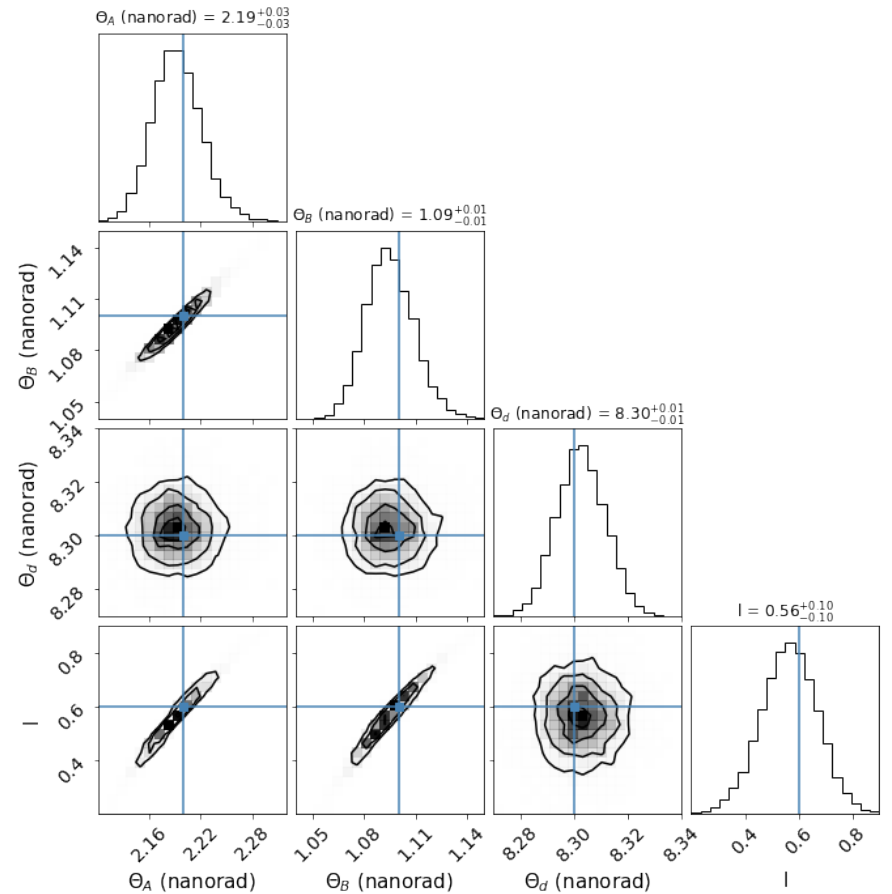

(b) $\mathrm{SNR}=1850$

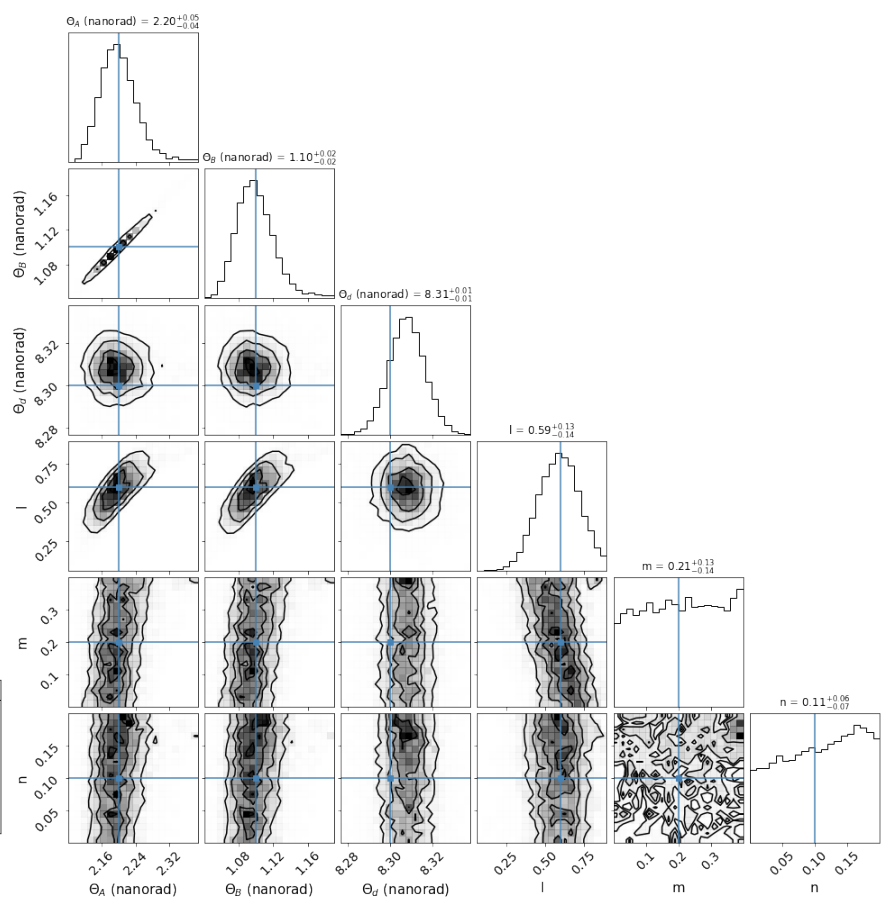

(d) $\mathrm{SNR}=1850$

Figure 11. Estimation of various numbers of parameters. The upper left panel estimates only the angular radii and the angular separation of the stars, with limb-darkening coefficients $l=0.6, m=0.2, n=0.1$ given. In the upper-right panel, $l$ is estimated while $m$ and $n$ are given. The lower-left corner plot estimates all parameters except $n$ and the lower right corner plot is for all parameter being estimated. The estimation of $m$ and $n$ is not successful because of their small contribution.

a total SNR of 350 suffices to estimate the angular radii of the stars and separation between the stars with reasonable accuracy. A higher total SNR of about 1800 is required to estimate the linear limb-darkening coefficient. The higher-order coefficients could not be recovered at this SNR, but that fact does not degrade the estimates of the other parameters.

The precision of parameter recovery improves with increasing total SNR, but it also has a strong dependence on the sampling of the $(u, v)$ plane. That is to say, even if two re- 
gions of the $(u, v)$ plane may contribute equally to the total SNR for given observing time, one of the regions may be much more useful for parameter estimation. We suggest that the derivatives of the visibility with respect to the parameters can help identify the most important parts of the interferometric plane. By concentrating on parts of $(u, v)$ plane so identified, we found that a total SNR of 30 was sufficient to recover the angular radii and separation.

These results indicate that binary stars are of considerable interest for current and future programs in intensityinterferometry. Individual targets, however, will pose their own special challenges. Three questions in particular need further study. One is how to allow for the finite size of the light buckets, which we have not considered at all in this work, but will certainly be an issue for wide binaries where the cosine modulation in the visibility can vary within the diameter of light collector. A second question that will arise is whether the $(u, v)$ sampling available from a particular observatory is sufficient to recover all the desired parameters. The pioneering work of Herbison-Evans et al. (1971) had the advantage that the light buckets were moveable on a circular railway track, making any desired $(u, v)$ up to a maximum radius possible. Current and planned intensity interferometers are restricted to what the rotation of the Earth provides. That said, the observable (eqn. 5) does not have an especially complicated dependence on $(u, v)$ - it is circular with a sinusoidal modulation along one direction — which suggests that a dense sampling of the interferometric plane is not essential for recovering the stellar radii and separation. The simulations shown in Figs. 7 and 8 further indicate that even if a region of the $(u, v)$ plane is unobserved, the parameters may nonetheless be recovered, because the functional form of the observable is known. This brings us to the third question needing further study: how robust is the parameter recovery to errors in the parameterisation itself? Gross errors like mistaking a three-star system for a binary are unlikely, because spectroscopy would provide an independent check of the binary nature of a system. But a poor limb-darkening model, or unmodelled rotational flattening or star-spots, are of some concern. Here the results shown in Fig. 11 are of interest. They show that parameters (in that case higherorder limb-darking parameters) which are not constrained at the available SNR get automatically marginalised out by the MCMC, and do not bias those parameters which can be inferred. This argues for retaining possibly-relevant parameters in a model even if they cannot be constrained but only marginalised over.

\section{DATA AVAILABILITY}

The simulated data and code to generate all the corner plots are included in the supplementary material.

\section{REFERENCES}

Abeysekara A. U., et al., 2020, Nature Astronomy

Acciari V. A., et al., 2020, MNRAS, 491, 1540

Baumgartner S., et al., 2020, MNRAS, 498, 4577

Berger J. P., et al., 2012, A\&ARv, 20, 53

Claret A., 2000, A\&A, 363, 1081

Claret A., Hauschildt P. H., 2003, A\&A, 412, 241
Diaz-Cordoves J., Gimenez A., 1992, A\&A, 259, 227

Docobo J. A., Campo P. P., Gomez J., Horch E. P., 2018, AJ, 156, 185

Dravins D., LeBohec S., Jensen H., Nuñez P. D., 2013, Astroparticle Physics, 43, 331

Foreman-Mackey D., Hogg D. W., Lang D., Goodman J., 2013, PASP, 125, 306

Hanbury Brown R., Twiss R. Q., 1957, Proceedings of the Royal Society of London Series A, 242, 300

Hanbury Brown R., Davis J., Lake R. J. W., Thompson R. J., 1974, Monthly Notices of the Royal Astronomical Society, 167, 475

Herbison-Evans D., Hanbury Brown R., Davis J., Allen L. R., 1971, MNRAS, 151, 161

Kieda D., et al., 2019, BAAS, 51, 275

Kjeldsen H., Bedding T. R., 1995, A\&A, 293, 87

Klinglesmith D. A., Sobieski S., 1970, AJ, 75, 175

Kopal Z., 1950, Harvard College Observatory Circular, 454, 1

Malvimat V., Wucknitz O., Saha P., 2014, MNRAS, 437, 798

Mandel L., Wolf E., 1965, Reviews of modern physics, 37, 231

Milne E. A., 1921, Monthly Notices of the Royal Astronomical Society, 81, 361

Nuñez P. D., Domiciano de Souza A., 2015, MNRAS, 453, 1999

Rivet J. P., et al., 2020, MNRAS, 494, 218

Rodrigues T. S., et al., 2017, MNRAS, 467, 1433

Russell H. N., 1928, AJ, 38, 89

Schwarzschild K., 1906, Nachrichten von der Gesellschaft der Wissenschaften zu Göttingen, Mathematisch-Physikalische Klasse, 1906, 41

Sing D. K., Désert J. M., Lecavelier Des Etangs A., Ballester G. E., Vidal-Madjar A., Parmentier V., Hebrard G., Henry G. W., 2009, A\&A, 505, 891

Stephenson C. B., Sanwal N. B., 1969, AJ, 74, 689

Thompson A. R., Moran J. M., Swenson G. W., 2017, Interferometry and synthesis in radio astronomy. Springer Nature

Tkachenko A., et al., 2016, MNRAS, 458, 1964

Zampieri L., Naletto G., Burtovoi A., Fiori M., Barbieri C., 2021, arXiv e-prints, p. arXiv:2107.05596 